\title{
Decision support for local environmental impact assessment
}

\author{
Daryl H. Hepting \\ Department of Computer Science, University of Regina, Regina, Saskatchewan, S4S 0A2, Canada
}

Received 11 September 2005; received in revised form 20 October 2005; accepted 21 December 2005

Available online 21 April 2006

\begin{abstract}
Sustainability has become a key concern for consumers and industry. However, coming to understand its implications in the context of daily life can be difficult. It may be hard to come to terms with the idea that traditional activities present environmental risks and may lead to real emergencies, manifested differently depending on local conditions. Everyone will be asked to do more but the move towards sustainability may require the total transformation of current attitudes and actions. This paper considers the role of informatics in responding to, and possibly averting, environmental emergencies by making information available to decision makers, whether they are individual consumers, institutional buyers, or emergency response personnel. If all choices can be presented within a spectrum of best and worst possible impacts for sustainability, decision makers can meaningfully weigh their options. Any evaluation on this basis is presently very difficult because one needs to independently assess and integrate possibly incomplete and conflicting information from a wide variety of sources. This paper describes the use of the cogito software, which embodies an approach for data access and presentation that is expected to provide significant benefits over existing web-based tools. The paper also considers the issues related to the collection and synthesis of disparate data.
\end{abstract}

(c) 2006 Elsevier Ltd. All rights reserved.

Keywords: Decision support systems; Environmentally preferable purchasing; User interfaces; Information visualization; Triple bottom line accounting; Triple top line design; Data set exploration

\section{Software availability}

Software: A binary distribution only, for Mac OS X or Windows XP.

Available: By request from the author.

Website: http://www.cs.uregina.ca/ hepting/cogito/ for more information.

\section{Introduction}

A recent OECD (Organization for Economic Cooperation and Development) report (OECD, 2002) describes the consumers in member countries as generally having a high level of activism with respect to green issues, but a relatively low willingness to pay. The report indicates this may be due to a general "fatigue" amongst consumers with respect to green

E-mail address: dhh@cs.uregina.ca products. There seems to be a decline in trust of nearly all sources of environmental information and an increase in confusion about prioritization of environmental goals and which actions can be most beneficial.

Many daily activities have the potential to lead to environmental emergencies. Where price is the prime consideration, consumers may be unwilling or unable to make connections between their actions and the consequences of those actions. McDonough and Braungart (2004) describe what may be a typical situation for manufacturers in the United States, who compete on this basis and source many cheap materials from Asia, "where occupational health standards are minimal, have been found to be carcinogenic. Globally sourced materials are rarely, if ever, assessed, so many 'lean-thinking' U.S. companies are applying efficiency measures to toxic materials. The result: cheap products, expensive waste management systems, and rising health care costs." Similarly, consumers may fail to consider that a large amount of household greenhouse gas emissions are related to direct energy consumption for food 
preparation, conservation, and transport. Life cycle analysis (Ciambrone, 1997) can help to point out these problems, but it may also be difficult to manage the resulting data, which might include provenance, ingredients, processing, and labour practices to name a few. The effect of each of these on individual performance may vary considerably. Basson and Petrie (in press) detail some of the problems of associating value judgements with performance information.

The 1994 Oslo Roundtable on Sustainable Production and Consumption (Oslo Roundtable on Sustainable Production and Consumption, Retrieved 28 February 2004) developed the following working definition of sustainable consumption, as:

an umbrella term that brings together a number of key issues, such as meeting needs, enhancing quality of life, improving resource efficiency, minimising waste, taking a life-cycle perspective and taking into account the equity dimension; integrating these component parts in the central question of how to provide the same or better services to meet the basic requirements of life and the aspiration for improvement, for both current and future generations, while continually reducing environmental damage and risks to human health.

While this definition focuses on reducing drawbacks, the ultimate goal is to focus on maximizing benefits. In discussing their approach to design, called cradle to cradle, McDonough and Braungart (McDonough and Braungart, 2002), present the notion that graduated filters can be applied in environmental decision making, allowing bad products to be rejected; inferior products to be tolerated until they can be replaced; average products to be optimized; and optimal products to be chosen outright. There is great potential for informatics to aid the consumer in dealing with these complexities of the information by providing tools that empower individuals to study and compare the data without becoming overwhelmed.

A framework for the unified presentation of this information is a first step in raising the awareness of environmental risks that are present within daily life. Ordinary citizens need to have this information, and have it presented in ways that allow them to make connections to their daily lives.

The remainder of the paper is organized as follows. Section 2 presents the decision objectives for the system, along with some motivation; Section 3 deals with the design of the framework in terms of interface, data, and presentation; and Section 4 discusses the use of the current software. The discussion in these sections is framed with an example of cleaning product information. Finally, Section 5 presents conclusions and directions for future work.

\section{Decision objectives}

The goal of this work is to create a web-based decision support tool to empower consumers with the ability to assess their purchases with respect to sustainability in their own local contexts. The web was chosen for the delivery platform because of its widespread use and flexibility (Smiatek, 2005 for example). If a consumer is interested in evaluating different product alternatives, say for cleaning products, that individual might first seek environmental information about a product, or a class of products, on the web. Fig. 1 lists the top three results from a "cleaning products environmental information" query. Of the information available through these URLs, much is inappropriate for direct application to the task of choosing amongst several cleaning products. The main exception is the Cleaning Products Pilot Project (CPPP) of the United States' Environmental Protection Agency (EPA)(\# 3 in the figure).

The EPA's CPPP was interesting for several reasons, mainly though because it is the most visible and the most accessible of such tools currently available. It provides three different attribute ranking tools (single, multiple, and weighted) to organize and evaluate a set of 29 industrial cleaning products. For each cleaning product, information with respect to the following eight environmental attributes is provided: skin irritation, food chain exposure (bioconcentration factor), air pollution potential (percentage of volatile organic compounds), contains fragrance, contains dye, product is a concentrate (reduced packaging), packaging is made of recyclable paper, and product minimizes exposure to concentrate. The information is always presented in tabular form. The difference between the three tools is in how they allow the user to organize and filter the products based on the attributes. There appear to be opportunities to improve the usability of these tools, but the choice of attribute names may play a large role in the accessibilty of the information.

The Office of Energy Efficiency within Natural Resources Canada provides a $\mathrm{CO} 2$ calculator on its website (NRCan, Accessed on 28 February 2004) to allow people to understand the harmful effects of idling vehicle engines. However, the results are available only for whole communities and only in terms tonnes of carbon dioxide emitted and dollars saved by conserving fuel. An individual consumer using this calculator can only guess at his or her own impact and may be even be disempowered to act alone. Although dollar savings are readily understood, carbon dioxide is expressed in tonnes and equivalently in gymnasiums. A more meaningful expression of carbon dioxide emissions might be in terms of the number of trees required to sequester them. After some searching on the web, one might be able to locate a resource to accomplish this translation from tonnes to trees needed (Roulet and Freedman, 1999). However, requiring the user to do this extra work of conversion is at odds with making the information accessible.

(1) EPP Cleaning Products, Environmentally Preferable Product Information: the Commonwealth of Massachusetts environmentally preferable products procurement program with information about environmentally preferable cleaning products (http://www.state.ma.us/osd/enviro/products/cleaning.htm).

(2) Sources of Indoor Air Pollution Organic Gases (Volatile Organic Compounds): indoor air quality information from the United States Environmental Protection Agency (http://www.epa.gov/iaq/voc.html).

(3) Environmentally Preferable Purchasing Cleaning Products Pilot Project Using the Environmental Attribute Matrix: cleaning products pilot project from the United States Environmental Protection Agency

(http://www.epa.gov/oppintr/epp/cleaners/select/using.html).

Fig. 1. Top three search results for a cleaning products environmental information query in google. 
From these examples, it becomes clear that sources for environmental information are of limited effectiveness when their presentation fails to consider how individual consumers might relate to the information. In the CPPP case, price and performance data that would be helpful for consumers is very difficult to obtain and requires localization. With respect to the carbon dioxide calculator, specific vehicle performance information would help to give consumers concrete information.

\section{Technical aspects}

Many of the details related to this approach have to do with the underlying data, which the consumer can access according to his or her own conceptual model.

\subsection{Conceptual models}

The construction of a uniform information resource for consumers must at first be concerned with how well consumers can access the information. In a study of the EPA's CPPP suite, participants were asked to rate the importance of each attribute used therein.

Within the 48 participants, taken from undergraduate computer science courses at the University of Regina, there was agreement about only three of the attributes (in order): skin irritation, air pollution potential, and food chain exposure. This result begs the question whether consumers are only able to use a small number of attributes in decision making. Perhaps the importance rating of attributes only illustrates consumers' connection to somewhat arbitrary labels.

At different times, people may have different conceptual models about what is important for their decision making. In order for a consumer to use a particular model, the data which supports it must be available. The naming of attributes and the connection of the attribute labels to real data determines the effectiveness with which that model can be used. The same data may also support many different conceptual models. For example, the three user types identified by Rizzoli and Young (1997) (environmental scientist, environmental manager, and environmental stakeholder) may each have different conceptual models. One of the keys for environmental stakeholders is to be able to develop their own models without undue influence from the other user types.

Ongoing research is required for the development of concise methods to reliably elicit conceptual models and extract attribute names from people who are not familiar with the particular, specialized vocabulary used by the other user types. Consistency in the conceptual model is important, to avoid interference with the decision-making process (Bouma et al., 2005). This effort is based on personal construct psychology (Kelly, 1955) and conjoint analysis (Myers, 1996), which are methods meant to focus on underlying ideas rather than what may seem like arbitrary syntax.

Each attribute has two or more values. If a product or service is identified as a collection of attributes, it is possible then to think of each product as being addressable by its value for each attribute. These addresses are part of the space of all available addresses, in the following form. For $N$ atttributes, each tuple of values is a distinct point in the product address space, as follows:

$\left\langle v_{1}, v_{2}, \ldots, v_{N}\right\rangle \in A_{1} \times A_{2} \times \ldots \times A_{N}$

A consumer's conceptual model may provide a great deal of resolution, or relatively little, depending to what degree alternatives are uniquely identified. If relatively few attributes are identified or articulated, many products may share the same address.

\subsection{Data collection}

In order to allow as much separation between the data and the conceptual models which they support, it is desirable to store as much basic data as possible and only transform it to fit a conceptual model when that data is accessed according to that model. The data presented in the EPA's CPPP provide a good starting point, but there is more information available than is contained in those attributes. Although manufacturers are not required by law to label their products with the ingredients used to produce these products, they must file a material safety data sheet (MSDS) for each product. The MSDS contains information about some of the ingredients, relevant safety information, health effects, first aid measures and precautions (ANPED, Accessed Fall 2004). Although consumers may find this information helpful, it is not easy to locate the desired MSDS, nor are the data sheets written in a manner that is accessible to most consumers. XML facilitates gathering of data by providing a medium for sharing relevant information, and supports the job of portraying data in a meaningful fashion. The use of XML also satisfies the need to properly document great quantities of data, a problem relevant to this endeavour. de Vos et al. (2001) further explain that "XML is accompanied by extensive technology infrastructure covering functions such as transformation, presentation, query, schema and exchange protocols... It imparts a degree of compatibility to different industry standards against the day when they come into contact with each other in the enterprise or on the public Internet." Using XML opens the door to information sharing among all interested parties, including government, industry, community organizations, and the general population. The related standards of RDF (Resource Description Framework) (W3C, Retrieved January 29, 2004) and XFML (eXchangeable Faceted Metadata Language (Dijck, Retrieved January 29, 2004) can provide the ability to maintain a central repository of metadata without requiring the data to also be maintained there, allowing greater freedom.

\subsection{Localization}

Attributes related to the price and performance of the cleaning products would add valuable information for a consumer. However, the presentation of this information presents certain challenges since it must be stored locally. Fig. 2 presents an overview of how this capability might be provided using 


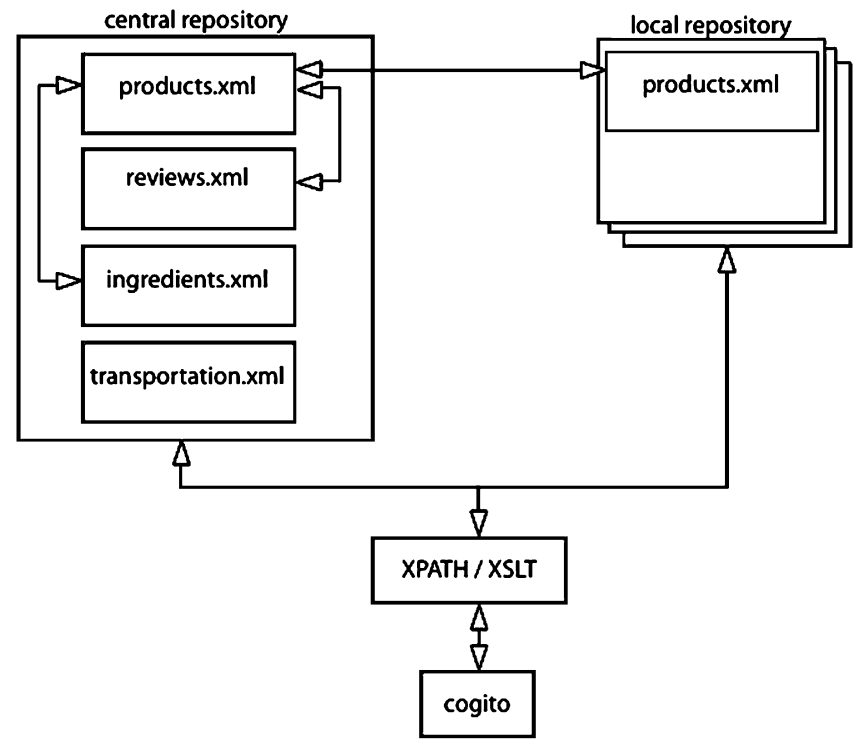

Fig. 2. Overview of information distribution within a unified framework. The central repository contains invariant product information and related data (including reviews from consumers). The local repositories contain the information that enables localization, according to price for example.

XPath and XSLT (Holman, 2001). This is the type of information with which a service like epinions.com (Shopping.com, Accessed on 28 February 2004) currently excels.

Local information is important in order to evaluate alternatives in the local context. However, global information is equally important. Because it can provide the larger perspective on what is being done around the world, it may spur interest in new products that are not yet locally available.

\subsection{Interaction}

The "augmented" model of human-computer interaction is used in this software, meaning that the software provides support enabling the user to exercise his or her judgement. These systems encompass a wide range of designs that are intended to allow the computer to support the users activities by working with the user. Augmented systems permit the user to start with an incomplete articulation of a problem and develop it interactively. Winograd and Flores (Winograd and Flores, 1985) further contend that such a definitive articulation, a priori, is not even possible. Wegner (1997) asserts that this interactive approach is also much more powerful.

Without going too far beyond the 29 products in the EPA CPPP example, an exhaustive search would be impractical. Recalling Eq. (1), it is possible to consider the space of all possible cleaning products, the size of which is determined as the cross product of all the attribute sizes, as a solution space (Simon, 1977), since the expectation exists that a solution to the current problem can be found amongst the combinations of attribute values. The notion of the product space is useful as a means to examine similar products whose addresses are close to one another.

In all cases when a user of computer software wants to find a solution, either he or she knows what is needed or wants to explore what is available. If the user is familiar with computers in general and the software specifically, then it may be an easy matter to specify a particular solution. However, this user may also be unaware of better solutions that exist outside of his or her experience, content with a local rather than global maximum. Exploration can be difficult, even for experienced users.

The parametric representation of the solution makes it well-suited to a genetic algorithm (Goldberg, 1989), but the standard implementations may be too automatic. The cogito system (Hepting, 2003), by contrast, relies on the user to direct the evaluation and exploration of successive query results until the desired information is found. Depicted in Fig. 3, a database may be viewed according to an attribute that has six distinct ranges of values. The user then sees six examples from this database, one from each of the six ranges. User selections at the display inform the next query to be made on the database.

\section{Example}

The interface depicted in Fig. 4 provides a user with a view of available cleaning product alternatives, organized in such a way as to provide an example of categorically different

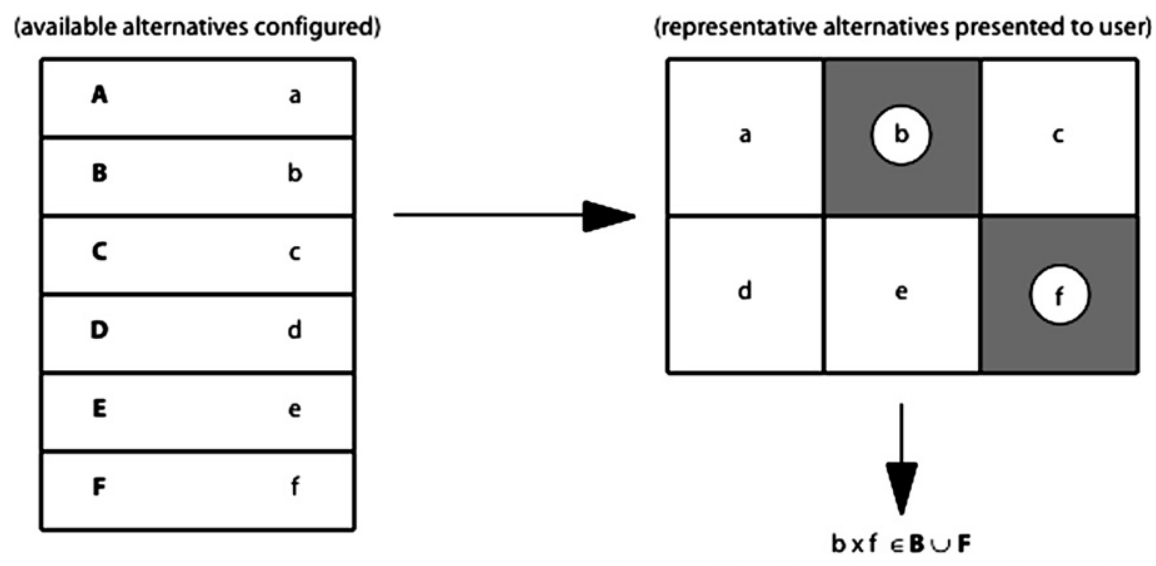

(user selection determines subspace for next iteration)

Fig. 3. Schematic view of the interface: the space of available alternatives is grouped according to user-specified criteria. Each group (A-F) has a representative element $(a-f)$ that is displayed to the user. The subspace for the next iteration is based on the user selection ( $b$ and $f$ ). 


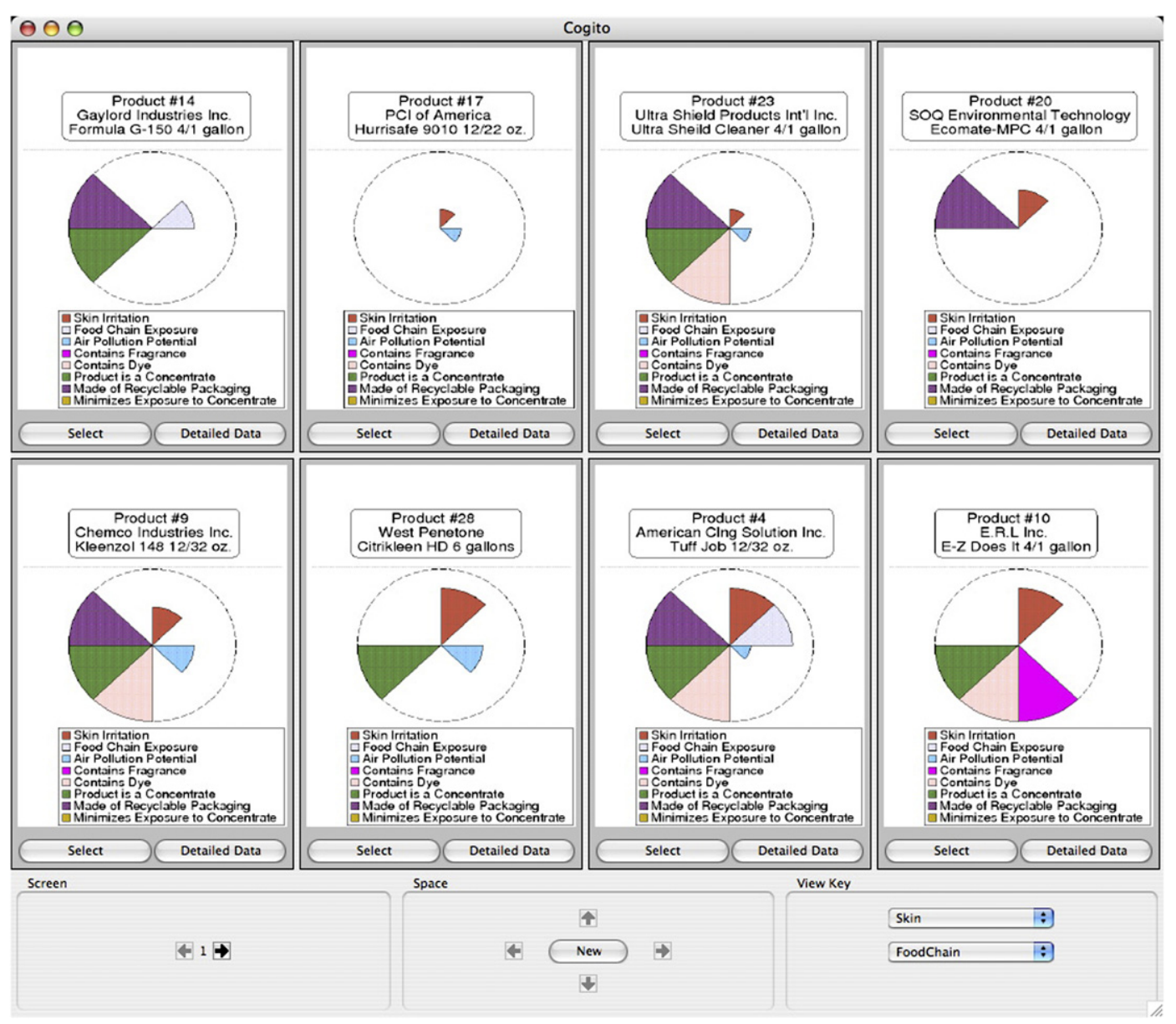

Fig. 4. A version of the cogito interface used to explore product data is depicted.

ones at any one time. Each cell in the display shows a visual representation of the product attribute values as a Nightingale Rose (Wainer, 1997), because of its better features for comparison. Like a pie chart it is circular, but whereas the pie chart varies the angle with the data but keeps radius constant, the Nightingale Rose maintains the angle and varies the radius with the data. In the figure, each rose is enclosed in a circle to relate each value to the maximum possible.

The data for the EPP wizard is continuous, but it is divided into different partitions. In all, there are about 5000 different combinations possible with these values. While choosing particular attributes in the interface, one can view examples of each partition and get a sense of what is possible. One can refine the options by making selections and refining them before executing a query. If not enough results are returned, a similarity metric can be applied to increase the number of matches.

\section{Conclusions and future work}

There is a great opportunity for informatics to contribute to sustainability and thereby minimize the risk of environmental emergencies. Dissemination of information about environmental risks, along with opportunities for action, will be an area of increasing importance. With greater awareness, consumers can seek to reframe the underlying goal from risk minimization to benefit maximization. The cogito model has shown great potential in other areas (Hepting and Gerhard, 2004; Hepting, 2003, 2002), and it will continue to be developed in this application to support exploration.

In addition to providing decision support for consumers, the collected and aggregated user interactions could be used to inform public policy and private investment: public policy can direct educational campaigns to increase awareness of factors not highly-rated by consumers and private investment can finance development of products where pronounced interest exists without any means of fulfillment. It is also useful to consider the opportunities for new products or the reasons why a portion of the product space contains no entries. One may be able to articulate the attribute values for an ideal cleaning product, but such an alternative may not exist within the system. Therefore the system should present the alternatives that best meet the present needs of the user, and allow the system to notify the user when better alternatives come along.

\section{Acknowledgements}

M. Buhler, O.Hoeber, Y. Liu, T. Maciag, M. McKague, and P. Schmiedge have contributed to this paper. This work was supported by the University of Regina Centre for Sustainable Communities and by the Science and Engineering Research Council. 


\section{References}

ANPED, 2004. Eliminating Barriers to Sustainable Production and Consumption, ANPED Issue Paper. <http://www.anped.org/PDF/SPAC\%20Issue\%20paper.pdf $>$ (accessed Fall).

Basson, L., Petrie, J.G. An integrated approach for the consideration of uncertainty in decision making supported by Life Cycle Assessment. Environmental Modelling and Software, in press.

Bouma, et al., 2005. Risk assessment and water management. Environmental Modelling and Software 20, 141-151.

Ciambrone, D.F., 1997. Environmental Life Cycle Analysis. Lewis Publishers, Inc.

Dijck, P.V. XFML Core - eXchangeable Faceted Metadata Language. Available from: $<$ http://www.xfml.org/spec/1.0.html > (accessed 29.1.04.).

Goldberg, D.E., 1989. Genetic Algorithms in Search, Optimization, and Machine Learning. Addison-Wesley, Reading, MA.

Hepting, D.H., 2002. Towards a visual interface for information visualization. In: Banissi, E. (Ed.), Proceedings of the Sixth International Conference on Information Visualization. IEEE Computer Society, pp. 295-302.

Hepting, D.H., 2003. Interactive evolution for systematic exploration of a parameter space. In: Dagli, C.H., et al. (Eds.), Intelligent Engineering Systems through Artificial Neural Networks, vol. 13. ASME Press, pp. $125-131$.

Hepting, D.H., Gerhard, D., 2004. Collaborative computer-aided parameter exploration for music and animation. In: Proceedings of the Second International Meeting on Computer Music Modelling and Retrieval, vol. 3101. LNCS.

Holman, G.K., 2001. Definitive XSLT and XPath. Prentice-Hall.

Kelly, G., 1955. The Psychology of Personal Constructs. Norton.

McDonough, W., Braungart, M., 2002. Cradle to Cradle: Remaking the Way We Make Things. North Point Press.
McDonough, W., Braungart, M., 2004. Beyond the triple bottom line: a new standard for 21st century commerce. Green Money Journal 13 (53). GreenMoneyJournal.com.

Myers, J.H., 1996. Segmentation and Positioning for Strategic Marketing Decisions. South-Western.

NRCan. Idle-free! Carbon dioxide calculator webpage from the Office of Energy Efficiency within Natural Resources Canada. $<$ http://oee.nrcan.gc.ca/ idling/calculator/ $\mathrm{CO} 2$ calculator.cfm $>$ (accessed 28.2.04.).

OECD, 2002. Towards Sustainable Household Consumption? Trends and Policies in OECD Countries. (OECD E-Book).

Oslo Roundtable on Sustainable Production and Consumption. Defining Sustainable Consumption. <http://www.iisd.ca/consume/oslo004.html> (accessed 28.2.04.).

Rizzoli, A.E., Young, W.J., 1997. Delivering environmental decision support systems: software tools and techniques. Environmental Modelling and Software 12 (2-3), 237-249.

Roulet, N.T., Freedman, B., 1999. What Trees Can Do to Reduce Atmospheric $\mathrm{CO}_{2}$. Tree Canada Foundation.

Shopping.com. <http://www.epinions.com/> (accessed 28.2.04.).

Smiatek, G., 2005. SOAP-based web services in GIS/RDBMS environment. Environmental Modelling and Software 20, 775-782.

Simon, H., 1977. Models of Discovery. Reidel.

de Vos, A., Widergren, S.E., Zhu, J., 2001. XML for CIM model exchange. In: Proceedings of Power Industry Computer Applications (PICA), pp. 31-37.

W3C. Resource Description Framework. <http://www.w3.org/RDF> (accessed 29.1.04.).

Wainer, H., 1997. Visual Revelations. Copernicus (Springer-Verlag).

Wegner, P., 1997. Why interaction is more powerful than algorithms. CACM 40, 80-91.

Winograd, T., Flores, C.F., 1985. Understanding Computers and Cognition. Ablex. 